\title{
Polarity and Non-polarity of Ionic Liquids viewed from the rotational dynamics of carbon monoxide
}

\author{
Y. Yasaka and Y. Kimura \\ Department of Molecular Chemistry and Biochemistry, Faculty of Science and Engineering, Doshisha University, \\ Kyotanabe, Kyoto 610-0321, Japan
}

Corresponding Authors

Yoshiro Yasaka $^{1}$ and Yoshifumi Kimura ${ }^{2}$

${ }^{1}$ Email : yyasaka@mail.doshisha.ac.jp, TEL +81-774-65-6562

${ }^{2}$ Email : yokimura@mail.doshisha.ac.jp, TEL +81-774-65-6561, FAX +81-774-65-6801 
Table S I. Samples

\begin{tabular}{|c|c|c|c|c|c|c|}
\hline \multirow[t]{2}{*}{ Solvent } & \multicolumn{2}{|c|}{$\begin{array}{l}\text { Gas phase conc. } \\
\text { / mM (/ bar) }\end{array}$} & \multicolumn{2}{|c|}{$\begin{array}{l}\text { Liquid phase conc. } \\
\text { / } \mathrm{mM}\end{array}$} & \multirow{2}{*}{$\begin{array}{c}{ }^{17} \mathrm{O} \text {-isotopic } \\
\text { purity }(\%)\end{array}$} & \multirow{2}{*}{$\begin{array}{c}\text { Ostwald } \\
\text { constant } \\
\text { of } \mathrm{CO}\end{array}$} \\
\hline & $\mathrm{C}^{17} \mathrm{O}$ & $\begin{array}{c}\mathrm{CO} \\
\text { (any isotope) }\end{array}$ & $\mathrm{C}^{17} \mathrm{O}$ & $\begin{array}{c}\mathrm{CO} \\
\text { (any isotope) }\end{array}$ & & \\
\hline$\left[\mathrm{C}_{2} \mathrm{C}_{1} \mathrm{im}\right]\left[\mathrm{NTf}_{2}\right]$ & $80(2)$ & $432(11)$ & 6.6 & 35 & 19 & 12 \\
\hline$\left[\mathrm{C}_{4} \mathrm{C}_{1} \mathrm{im}\right]\left[\mathrm{NTf}_{2}\right]$ & $84(2)$ & $521(13)$ & 7.2 & 45 & 16 & 12 \\
\hline$\left[\mathrm{C}_{8} \mathrm{C}_{1} \mathrm{im}\right]\left[\mathrm{NTf}_{2}\right]$ & $59(1.5)$ & 385 (15) & 6.1 & 39 & 15 & 10 \\
\hline$\left[\mathrm{P}_{4441}\right]\left[\mathrm{NTf}_{2}\right]$ & $58(1.5)$ & $390(10)$ & 6.4 & 43 & 15 & 9 \\
\hline$\left[\mathrm{P}_{8888}\right]\left[\mathrm{NTf}_{2}\right]$ & $74(2)$ & 657 (16) & 6.6 & 60 & 11 & 11 \\
\hline$n$-hexane & $99(2)$ & $602(15)$ & 34 & 212 & 16 & 2.9 \\
\hline$n$-dodecane & $280(7)$ & $1720(43)$ & 52 & 325 & 16 & 5.4 \\
\hline squalane & $163(4)$ & $1050(26)$ & 19 & 123 & 15 & 8.6 \\
\hline
\end{tabular}


Table S II $\quad T_{1}$ (in ms) of CO in ILs and alkanes.

\begin{tabular}{|c|c|c|c|c|c|c|c|c|}
\hline$T /{ }^{\circ} \mathrm{C}$ & $\begin{array}{c}{\left[\mathrm{C}_{2} \mathrm{C}_{1} \mathrm{im}\right]} \\
{\left[\mathrm{NTf}_{2}\right]}\end{array}$ & $\begin{array}{c}{\left[\mathrm{C}_{4} \mathrm{C}_{1} \mathrm{im}\right]} \\
{\left[\mathrm{NTf}_{2}\right]}\end{array}$ & $\begin{array}{c}{\left[\mathrm{C}_{8} \mathrm{C}_{1} \mathrm{im}\right]} \\
{\left[\mathrm{NTf}_{2}\right]}\end{array}$ & $\begin{array}{l}{\left[\mathrm{P}_{4441}\right]} \\
{\left[\mathrm{NTf}_{2}\right]}\end{array}$ & $\begin{array}{l}{\left[\mathrm{P}_{8888}\right]} \\
{\left[\mathrm{NTf}_{2}\right]}\end{array}$ & $n$-hexane & n-dodecane & Squalane \\
\hline-80 & & & & & & $241(4)$ & & \\
\hline-40 & & & & & & $319(7)$ & & \\
\hline-10 & & & & & & & $221(5)$ & $25.2(4)$ \\
\hline 0 & & & & & & & $250(5)$ & $46(1)$ \\
\hline 12 & & & & & & & $266(5)$ & $79(1)$ \\
\hline 20 & & & & & & $356(3)$ & & \\
\hline 22.5 & & $25(2)$ & & & & $360(14)$ & & \\
\hline 23 & $29(1)$ & & $20.5(5)$ & $20(1)$ & $31(1)$ & $349(4)$ & & $113(2)$ \\
\hline 25 & & & & & & & 297(9) & \\
\hline 30 & & & & & & $342(4)$ & & \\
\hline 40 & $45(1)$ & $42(2)$ & $41(2)$ & $36(1)$ & $54(1)$ & $346(4)$ & $319(7)$ & $178(3)$ \\
\hline 50 & & & & & & $349(6)$ & & \\
\hline 60 & $78(2)$ & $66(2)$ & $72(2)$ & $62(2)$ & $92(4)$ & $340(5)$ & $327(12)$ & $239(4)$ \\
\hline 80 & $104(3)$ & $97(2)$ & $108(4)$ & 101(3) & $129(3)$ & & $330(12)$ & $283(6)$ \\
\hline 100 & $133(5)$ & & $138(8)$ & $135(3)$ & $174(6)$ & & $335(8)$ & 291(7) \\
\hline 120 & $170(6)$ & $193(8)$ & 191(5) & $171(7)$ & $206(6)$ & & & $308(8)$ \\
\hline 140 & & & & $217(13)$ & $254(9)$ & & & $327(7)$ \\
\hline
\end{tabular}


Table III $\tau_{2 \mathrm{R}}$ (in ps) of CO in ILs and alkanes.

\begin{tabular}{|c|c|c|c|c|c|c|c|c|}
\hline$T /{ }^{\circ} \mathrm{C}$ & $\begin{array}{c}{\left[\mathrm{C}_{2} \mathrm{C}_{1} \mathrm{im}\right]} \\
{\left[\mathrm{NTf}_{2}\right]}\end{array}$ & $\begin{array}{c}{\left[\mathrm{C}_{4} \mathrm{C}_{1} \mathrm{im}\right]} \\
{\left[\mathrm{NTf}_{2}\right]}\end{array}$ & $\begin{array}{c}{\left[\mathrm{C}_{8} \mathrm{C}_{1} \mathrm{im}\right]} \\
{\left[\mathrm{NTf}_{2}\right]}\end{array}$ & $\begin{array}{l}{\left[\mathrm{P}_{4441}\right]} \\
{\left[\mathrm{NTf}_{2}\right]}\end{array}$ & $\begin{array}{l}{\left[\mathrm{P}_{8888}\right]} \\
{\left[\mathrm{NTf}_{2}\right]}\end{array}$ & $n$-hexane & n-dodecane & $\begin{array}{c}\text { Squalan } \\
\mathrm{e}\end{array}$ \\
\hline-80 & & & & & & $0.233(4)$ & & \\
\hline-40 & & & & & & $0.176(4)$ & & \\
\hline-10 & & & & & & & $0.254(6)$ & $2.23(3)$ \\
\hline 0 & & & & & & & $0.224(4)$ & $1.22(3)$ \\
\hline 12 & & & & & & & $0.211(4)$ & $0.71(1)$ \\
\hline 20 & & & & & & $0.158(1)$ & & \\
\hline 22.5 & & $2.2(2)$ & & & $1.81(6)$ & $0.156(6)$ & & \\
\hline 23 & $1.94(6)$ & & $2.74(7)$ & $2.8(1)$ & & $0.161(2)$ & & $0.50(1)$ \\
\hline 25 & & & & & & & $0.189(6)$ & \\
\hline 30 & & & & & & $0.164(2)$ & & \\
\hline 40 & $1.25(3)$ & $1.3(1)$ & $1.38(6)$ & $1.6(1)$ & $1.03(2)$ & $0.158(2)$ & $0.176(4)$ & $0.32(1)$ \\
\hline 50 & & & & & & $0.160(3)$ & & \\
\hline 60 & $0.72(2)$ & $0.85(2)$ & $0.78(2)$ & $0.90(3)$ & $0.61(3)$ & $0.165(2)$ & $0.172(6)$ & $0.23(1)$ \\
\hline 80 & $0.54(2)$ & $0.58(1)$ & $0.52(2)$ & $0.56(2)$ & $0.43(1)$ & & $0.170(6)$ & $0.20(1)$ \\
\hline 100 & $0.42(2)$ & & $0.41(2)$ & $0.42(1)$ & $0.32(1)$ & & $0.167(4)$ & $0.19(1)$ \\
\hline 120 & $0.33(1)$ & $0.29(1)$ & $0.294(7)$ & $0.33(1)$ & $0.27(1)$ & & & $0.18(1)$ \\
\hline 140 & & & & $0.26(1)$ & $0.22(1)$ & & & $0.17(1)$ \\
\hline
\end{tabular}


Table S IV. Temperature dependence of the viscosity (mPa s) of $\left[\mathrm{P}_{4441}\right]\left[\mathrm{NTf}_{2}\right],\left[\mathrm{P}_{8888}\right]\left[\mathrm{NTf}_{2}\right]$ and $\mathrm{Cs}\left[\mathrm{NTf}_{2}\right]$.

\begin{tabular}{rccc}
\hline$T / \mathrm{K}$ & {$\left[\mathrm{P}_{4441}\right]\left[\mathrm{NTf}_{2}\right]$} & {$\left[\mathrm{P}_{8888}\right]\left[\mathrm{NTf}_{2}\right]$} & $\mathrm{Cs}\left[\mathrm{NTf}_{2}\right]$. \\
\hline 293.15 & 284 & 429 & \\
313.15 & 92.3 & 150 & \\
333.15 & 38.5 & 63 & \\
353.15 & 18.9 & 30.9 & \\
373.15 & 10.8 & 17.4 & \\
393.15 & 6.9 & 10.6 & 28.8 \\
413.15 & 4.7 & 6.8 & 28.7 \\
433.15 & 3.4 & 4.8 & 18.8 \\
453.15 & 2.7 & 3.6 & \\
\hline
\end{tabular}

Table S V. Parameters of the viscosities obtained by the fit to the following equation.

$$
\eta=\eta_{0} \exp \left(\frac{B}{T-T_{0}}\right)
$$

\begin{tabular}{lccc}
\hline & $\eta_{0} / \mathrm{mPa} s$ & $B / \mathrm{K}$ & $T_{0} / \mathrm{K}$ \\
\hline$\left[\mathrm{P}_{4441}\right]\left[\mathrm{NTf}_{2}\right]$ & 0.0680 & 1038 & 168.9 \\
{$\left[\mathrm{P}_{8888}\right]\left[\mathrm{NTf}_{2}\right]$} & 0.0325 & 1484 & 137.0 \\
\hline
\end{tabular}

Table S VI. Parameters obtained by the fit of $\tau_{2 \mathrm{R}}$ to equation (8)

\begin{tabular}{|c|c|c|c|c|c|}
\hline & {$\left[\mathrm{C}_{2} \mathrm{C}_{1} \mathrm{im}\right]\left[\mathrm{NTf}_{2}\right]$} & {$\left[\mathrm{C}_{4} \mathrm{C}_{1} \mathrm{im}\right]\left[\mathrm{NTf}_{2}\right]$} & {$\left[\mathrm{C}_{8} \mathrm{C}_{1} \mathrm{im}\right]\left[\mathrm{NTf}_{2}\right]$} & {$\left[\mathrm{P}_{4441}\right]\left[\mathrm{NTf}_{2}\right]$} & {$\left[\mathrm{P}_{8888}\right]\left[\mathrm{NTf}_{2}\right]$} \\
\hline$\tau_{2 \mathrm{R}}{ }^{0}(\mathrm{ps})$ & 10.20 & 7.96 & 5.40 & 3.11 & 1.49 \\
\hline$P$ & 0.77 & 0.73 & 0.69 & 0.57 & 0.49 \\
\hline
\end{tabular}


Figure S1. The fitting of the rotational relaxation time to equation (8).

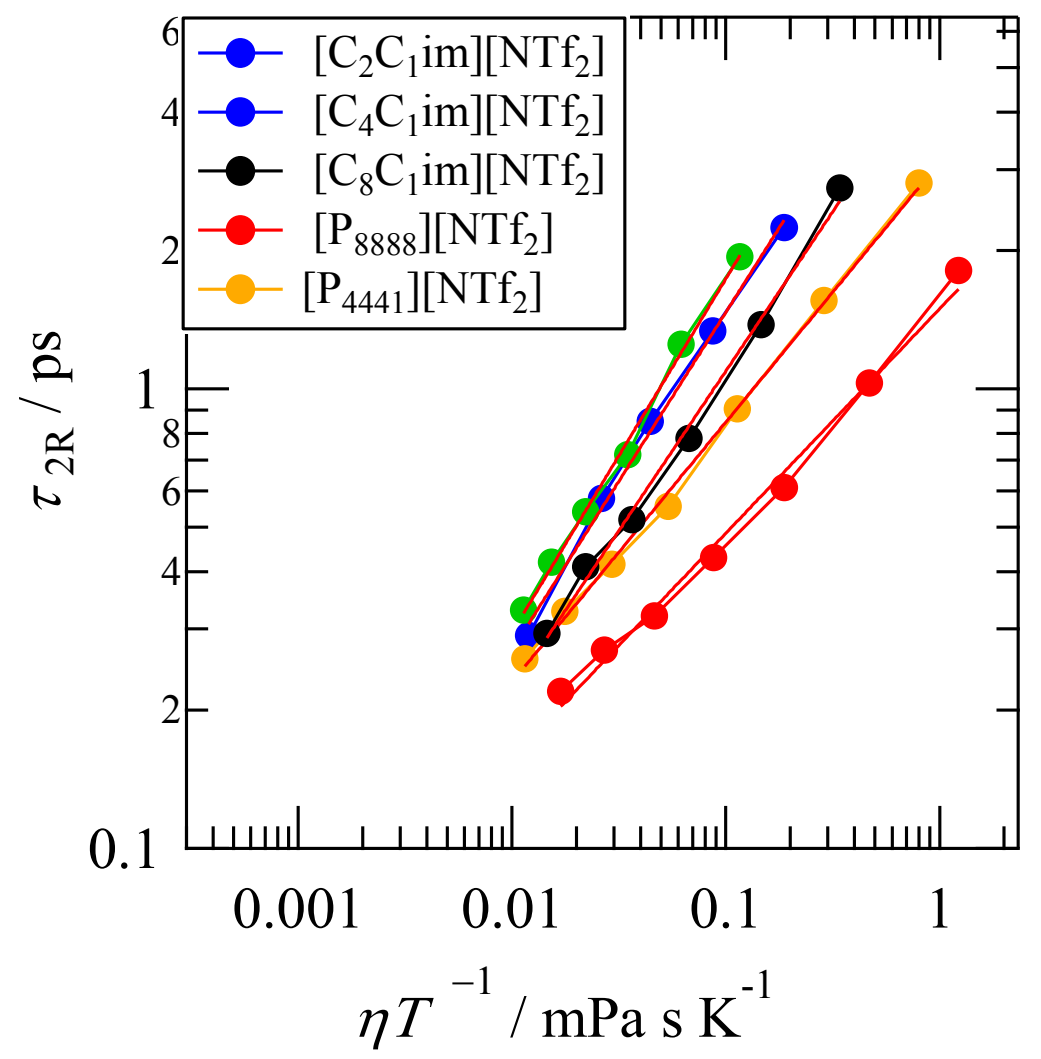

